\title{
Die anthropologische Differenz der Medien. Wissenschaft und Phantasma
}

Unauffällige und sehr kleine Gesten sind es, eine Fingerspitze, die die Lippe berührt, ein ganz vorsichtiges Zurückstreichen der Haarsträhne, zwei Finger einer Hand, die die andere suchen, kaum spürbare Berührungen des eigenen Körpers, an denen die Leute auf sich selbst und das sensomotorisches Band aufmerksam werden, das den für ein Menschliches konstitutiven Zusammenhang von Leib, Wahrnehmung und Gestalt bestätigt. Reveries, Tagträume, Träumereien kaum unterhalb des Bewusstseins sind es, die der Menschenmacher Dr. Robert Ford den Algorithmen seiner künstlichen Wesen als Mikrogesten einprogrammiert. Es zeigt sich, dass den Figuren damit blitzartig Bilder der Erinnerung durch die Vorstellung schießen, ein Vorgang, den die Zuschauenden als Montage von flash-frames auf dem Bildschirm sehen. Solche Erinnerungsfetzen führen im Einklang mit jeder Erinnerungstheorie des 20. Jahrhunderts dazu, dass das Verhalten der Androiden beginnt, Inkonsistenzen aufzuweisen, dass sie ihre implementierten Routinen und Narrative verlassen, dass sie Bewusstsein entwickeln. ${ }^{1}$ Mit diesem ausgestattet werden sie sich schließlich gegen ihre Schöpfer auflehnen. So erzählt es die Serie Westworld, ${ }^{2}$ die sich selbst aus einer Art medienphilologisch inspiriertem Erinnerungsblitz entwickelt: in Dialogen und Einstellungen, in der Inszenierung des Labors ebenso wie im Verhalten der Androiden erinnert die Serie filmisch implizit an die Geschichte des berühmtesten unter den künstlichen Geschöpfen, an Frankensteins Monster, das seinem Schöpfer begegnen will, um ihn zu erledigen.

Frankensteins Monster in seinen behäbigen Schritten und der schlechten hapto-motorischen Rückkopplung, mit der es Blumen und Mädchen viel zu grob packt, verletzt und vernichtet, hat, wie sich gerade im Vergleich der Verfilmung von James Whale $^{3}$ mit dem Roman Mary Shelleys beobachten lässt, den Medientransfer einer Differenz überstanden: der Unterscheidung von Mensch und Maschinenwesen im Übergang von der Bücher- in die Kinofantasie. Die Büchermenschen trennte von ihren so sehr ähnlichen Brüdern und Schwestern, den Androiden und Androidinnen, vor allem eins: Dem Monster fehlte eine Seele. James Whales Kinokerl aber zeigt deutlich, inwiefern sich ein filmisches Monster von allen literarischen AndroidenFantasien unterscheidet: Der literarische Doppelgänger oder Androide, wie er um 1800 ins Leben gerufen wird, gehorcht den Gesetzen der symbolischen Schrift, die nur

1 Das gilt für die Psychoanalyse in ihrer klassischen oder strukturalen Variante ebenso wie für die philologisch motivierte Erinnerungstheorie von Aleida und Jan Assmann. Vgl. Erik Porath: Gedächtnis des Unerinnerbaren. Philosophische und medientheoretische Untersuchungen zur Freudschen Psychoanalyse, Bielefeld 2005.

2 Jonathan Nolan: Westworld, Staffel 1 (USA: Bad Robot Productions/Kilter Films/Warner Bros. Television/Jerry Weintraub Productions 2016), TV-Ausstrahlungen auf HBO seit 10/2016, 10 Folgen, 60_90 min.

3 James Whale: Frankenstein (USA: Universal 1931), 70 min. 
ein „individuelles Allgemeines“ ${ }^{4}$ abbilden kann. Erst die analogen technischen Medien des späten 19. Jahrhunderts - Fotografie, Film und Phonographie - können Singularitäten abbilden: spezifische Bewegungen eines Körpers, einer Mimik oder einer Stimme, deren Spuren, mit wieder anderen Medientechniken, als unverwechselbare identifiziert werden. Was ein Mensch sein will, kommt um das Wissen und die Logik solcher Medienanordnungen nicht herum. Die Serie Westworld wirft gegenwärtig für das 21. Jahrhundert die Frage auf, welche Medien und Netzwerke bestimmen, was Menschen sind und wie sie sich von ihren androiden Doppelgängern unterscheiden könnten. Dazu kehrt auch die Serie noch einmal zurück zur Genealogie künstlicher Menschen in wissenschaftlichen Laboratorien und damit zur alten Kinogeschichte.

Im Kino werden Monstren, diese buchstäblich zu zeigenden und wahrzunehmenden Dinger, zu Androiden, die im Austausch mit der Welt der Dinge und Reize, in die sie gesetzt wurden, funktionieren. Ihre Gefühle erweisen sich, sichtbar für alle Zuschauer und Zuschauerinnen, als beständiges Arbeiten an der Rückkopplung des Sensomotorischen; auf diesem Weg des kybernetischen Lernens werden sie menschlicher. Schon der Golem in Paul Wegeners Film von 1921 wird damit enden, dass der grobe Kerl der kabbalistischen Mystik am Ende des Films Blumen und Mädchen vorsichtig anfassen kann und träumend seinen eigenen Berührungen nachhängen. Gefühle, das wissen Filmschauspielende und Regieführende ebenso wie das Publikum, sind nichts anderes als allmähliche Verfeinerungen der Rückkopplung von Sinnlichkeit und Bewegung. Entsprechend zeigt sich der Kinomensch durch ein sichtbares Verhalten, in dem Leib, Gestalt und Wahrnehmung als Sensomotorik mehr oder weniger grob, mehr oder weniger fein rückgekoppelt sind. Als Form einer solchen energetischen zirkulär-kausalen Rückkopplung zwischen Kino, Welt und Körpern hat Edgar Morin 1956 das Kino beschrieben: „Ein Werk der Phantasie ist eine radioaktive Batterie von Projektion - Identifikation. [...] Ein wirklich energetischer Stromkreis ermöglicht es, Partizipation in starker Dosierung zu vergegenständlichen, um sie dem Publikum wieder zuzuführen. ${ }^{55}$ Spätestens seit den fünfziger Jahren wird das Subjekt als Hybrid aus Kino und Mensch vorgestellt.

Westworld verhandelt ein wiederum neues Menschenbild, in dem die alten Kinomenschen und -monster verabschiedet werden zugunsten eines wirklich kybernetischen Menschen, der zwischen medizinischen Laboratorien, Experimenten der Computersimulation und alltäglichen medialen Praktiken entsteht. Deutlicher als im

4 Friedrich Kittler: Romantik - Psychoanalyse-Film. In: ders. (Hg.): Draculas Vermächtnis. Technische Schriften, Leipzig 1993, S. 81-104, S. 86.

5 Vgl. Edgar Morin: Der Mensch und das Kino. Eine anthropologische Untersuchung, Stuttgart 1958, S. 122. 
Film gleichen Namens aus dem Jahr $1973^{6}$ bindet die neuere Serie ihre Erzählung an Vorstellungen vom Menschen, die ihre Genealogie in Phantasmen medizinischer Bildgebungsverfahren und deren Material Cultures ebenso haben wie in Verfahren und Formaten des sogenannten postkinematografischen Kinos mit seinen neuen und skalenfreien Oberflächen. ${ }^{7}$ Die neue Serie ist nicht mehr nur, wie Michael Crichtons Original, ein amusement park des Wilden Westens, in dem Mord, Totschlag, Erniedrigung und Vergewaltigung der lokalen Bevölkerung allen zahlenden Gästen offenstehen. Sie positioniert sich vielmehr exakt zwischen Kulturtechniken des Abenteuerparks und jenen des Videospiels. Deshalb heißen die Indigenen auch hosts, halbwegs zwischen Datenspeichern und Gastgebern. In den hosts ist die Rückkopplung zwischen Taktilem und Sensorischem zum Automatismus des Verhaltens geworden. Das ist nichts Neues. Vergnügt üben schon Kleinkinder die spezifische Körpertechnik der Integration von Auge und Hand oder Auge und Daumen an Videospielen ein. So können sie später, wenn sie zahlenden Zugang zur Welt haben und nicht zum prekären Volk der Benutzbaren gehören, mit demselben Verhalten an entsprechenden Schnittstellen in den amusement park Erde intervenieren, als Managerinnen oder Krankenpfleger, als Meeresbiologinnen oder Piloten, können ihn entweder retten oder vernichten. ${ }^{8}$ Für die Indigenen dieser Welt, die unfreiwilligen hosts der reicheren Eliten, macht das freilich kaum einen Unterschied.

Als Trainingscamp etabliert Westworld mithin ein Szenario im doppelten Sinne von Drehbuch und filmischem Szenenbild, ein zugleich überschaubares und begehbares Modell, das aus einem leicht antiquierten Fundus alter Westernfilme besteht. Damit können sich auch die alten Kinomenschen aus der alten Welt an das erinnern, was sie selbst einmal im Kino oder im Fernsehen sahen. Eine Immersion ins Fiktive, die im alten Medium Buch noch metaphorisch beschrieben und im Dispositiv Kino im Imaginären bewirkt werden musste, ist, so die Annahme der Serie, unter Bedingungen beweglicher Oberflächen omnipräsenter Bilder als Touchscreens stets bereits als Verhalten realisiert. Was wir bildlich vor uns haben, als Spiel, Film oder amusement park ist niemals nur Schauspiel einer Welt. Nicht wir verfügen, sie per Monitor betrachtend, über diese Bildwelten, sondern wir sind deren Unterworfene. Ob als silly oder serious game konzipiert, unser Verhalten als senso- oder hapto-motorischer Zugang zur Welt - oder wie Maurice Merlau-Ponty deutlicher anmerkt: unser Verhalten als Sein-zurWelt - folgt Algorithmen, die meistens andere schreiben und vertreiben. Auch wenn in

\footnotetext{
6 Michael Crichton: Westworld (USA: MGM 1973), 89 min. Im Folgenden ist mit Westworld stets die neue Serie gemeint.

7 Vgl. Steven Shavir: Post Cinematic Affect, Winchester/Washington 2010.

8 Michel Serres: Erfindet euch neu! Eine Liebeserklärung an die vernetzte Generation, Berlin 2013.
} 
der Westworld zunächst die Erfahrung und Koordination eines relativ überschaubaren Milieus aufgerufen wird, auf das die Gäste singulär, konkret und mikrostrategisch mit kleinsten Bewegungen reagieren müssen, entspricht das genau dem Lernen in der Welt, die wir die wirkliche nennen: an den Schnittstellen von Computern, gestalteten Bildwelten und erhöhten Wahrnehmungskapazitäten bildet sich das Verhalten des gegenwärtig neuen Menschen wie in einem Spiel. Daher hat Claus Pias vorgeschlagen, „versuchsweise einmal alle Computeranwendungen als Spiele zu thematisieren, die ,im wirklichen Leben im Gange sind“ und diese zum Grund von Spieltheorie zu machen". ${ }^{9}$ Westworld thematisiert Verhalten entsprechend als Schauspieltheorie: eine neue Vernetzung der Körper, die alte Gewaltverhältnisse mitschleppt.

Die neuen Menschen haben, darauf insistiert der Vorspann der Serie, ihre Genealogie in mechanischen, physiologischen, materialen und elektronisch rückkoppelnden Forschungen und Wissensformationen, dem ganzen Arsenal westlicher Laboratorien des 19. und des 20. Jahrhunderts. Für die antiquierten analogen Kinomenschen ist unheimlich, wie die heutigen poucettes, ${ }^{10}$ Däumlinge der manuell-gesteuerten Schnittstellen, nach Michel Serres' Diagnose, bei lebendigem Leibe von einem neuen Bewusstsein von außen ergriffen werden, als quasi reflektierte Innenansicht gegenwärtig herrschender Mediensysteme. ${ }^{11}$ Dass auch die alten Kinomenschen von außen und nicht mehr von einer internen Seele gesteuert waren, kommt erst angesichts der digitalisierten Netzwerk-Menschen unerwartet in den Blick. Die Erfahrung, als revery, Tagtraum, Träumerei, in einer schönen Trance, an Computer und Monitore gefesselt zu sein, mit einem technisch integrierten Bewusstsein verbunden, das als Teil des eigenen nur bewusst wird, wenn sich jemand die Augen reibt, über die Lippen streicht, die Haare zurückschiebt, zeigt sich anachronistisch immer nur an den anderen, der anderen Medien-Bewusstseins-Generation. So alt die Kinomenschen für die poucettes sind, so seltsam sind die für jene. Die Serie Westworld organisiert eine Begegnung beider, an der Schnittstelle von Mediengenerationen, als Spiegelbild von historisch unterschiedlichen Formen des Imaginären. Damit wird jedes Ich als immer von Außen her, aus medialisierten Gestalten generiertes erkennbar: als Anderes.

9 Claus Pias: Wirklich problematisch. Lernen von ,frivolen Gegenständen‘. In: ders., Christian Holtorf (Hg.): Escape! Computerspiele als Kulturtechnik, Weimar/Wien 2007, S. 255-269, S. 269.

10 Serres (s. Anm. 8), S. 23.

11 Vgl. Friedrich Kittler: Die Welt des Symbolischen, eine Welt der Maschine. In: ders. (Hg.): Draculas Vermächtnis (s. Anm. 4), S. 58-80, S. 61. 


\section{Verhalten als Austausch von Information und Störung}

Ob er die ganze Nacht hier draußen gewesen sei, fragt Dolores Abernathy ihren Vater Peter, als sie ihn morgens selbstvergessen auf der Veranda sitzend vorfindet. In der Serie Westworld wird mit dieser Frage ein Verhaltens-Loop zurückgesetzt, der das Narrativ der Figur Dolores immer wieder von vorne starten lässt. ${ }^{2}$ Weil die Narration außer Kontrolle gerät, müssen die Ingenieure immer wieder eingreifen und das Verhalten der hosts zurücksetzen. Bereits in der Pilotfolge geschieht dieser Reset mehrmals, zuerst eben auf der Veranda, jenem klassischen Ort des homesteaders im Western, an dem der Blick auf die Wildnis draußen gerichtet wird. Aber es ist nicht das abwesende Träumen des Vaters oder eine Inkonsistenz seiner Aussagen, die Tochter Dolores an dieser Stelle stutzig machen, sondern das repetitive Verhalten ihres Vaters: er ist offenbar neben der Spur, out of order. Flüsternd und zuckend sitzt der alte Mann in seinem Schaukelstuhl und versucht sich zu artikulieren. ${ }^{13}$ Der gerade aus seiner Programmierung laufende host Peter kennt keine kohärente Innerlichkeit, er ist weder Monade noch Descart'sches cogito, das sich in der Trennung von cogitans und extensa wieder erkennen könnte. Vielmehr ist die Struktur des im Übrigen vom Anblick einer alten Fotografie gestörten Peters als Verhalten ,zugleich von außen für den Beobachter wie auch von innen für den Akteur sichtbar" ${ }^{14}$ Dolores nimmt die Verstörung des Vaters als Veränderung seines Körperschemas wahr. Ein Konzept einer Introspektion zum Verstehen des Anderen ist nicht mehr nötig. Der malfunctioning host auf dem Schaukelstuhl flüstert seiner noch regelhaft funktionierenden host daughter die scheinbare Erklärung - inklusive Warnung - ins Ohr. Die geflüsterte Nachricht können die Zusehenden nicht teilen, dafür aber unmittelbar teilhaben am Verhalten der hosts. Ein Verhalten ist, so MerleauPonty, „nicht mehr bloß ,visuelle Gegebenheit', deren psychologische Bedeutung wir in unserer inneren Erfahrung aufzuspüren hätten; der Psychismus des Anderen wird ein unmittelbarer Gegenstand “. ${ }^{15}$ Auch der Phänomenologe kam zu dieser Annahme mit Blick auf einen nicht nach Norm funktionierenden Körper: dem Phänomen des Phantomgliedes. Dieses Phänomen hatte in den vierziger Jahren des 20. Jahrhunderts, als Merleau-Ponty die Phänomenologie der Wahrnehmung schrieb, Konjunktur. Das Phantom des Anderen als konstitutiv für alles Verhalten zu begreifen ist ein Topos nicht nur französischer Nachkriegsphilosophie. Der Spielplatz von Westworld wendet diese Verstrickung ins Virtuelle unserer Gegenwart. Hier erkennen die Schöpfer der künstlichen Welten, die Mitarbeiter und Mitarbeiterinnen der Delos Company,

12 Nolan (s. Anm. 2), Staffel 1, Episode X.

13 Nolan (s. Anm. 2), Staffel 1, Episode I, TC: 00:45:10.

14 Maurice Merleau-Ponty: Die Struktur des Verhaltens, Berlin/New York 1976, S. 256.

15 Maurice Merleau-Ponty: Phänomenologie der Wahrnehmung, Berlin/New York 1966, S. 82. 
unmittelbar am Verhalten der hosts, dass diese sich ihrer programmierten Identitäten entziehen wollen. Verhalten sich die hosts nicht nach den ihnen zugeschriebenen Narrativen, wird das Programm gestoppt - Standbild - und der Tatort in eine Szenerie forensischer Spurensicherung verwandelt.

Bereits die Chronofotografien von Étienne-Jules Marey folgen diesem Changieren von Bewegung und Stillstellung, ganz genauso wie später die konstruktivistischen Filme der russischen Avantgarde. Sistierung und Bewegung des Bildlaufs, Analyse und Synthese des Bewegungseindrucks, wurden zugunsten der Erziehung eines neuen Menschen in Anschlag gebracht. Dessen Selbsterkenntnis und letztlich Wahrheit im anthropologischen, biologischen und psychologischen Sinn - wurde erst mit der Evidenz und der Wahrnehmung durch und dank der Kamera angenommen. Dziga Vertovs celovek's kinoapparatom, dessen Titel als Mann mit der Kamera, aber ebenso als „Mensch, wie der Kinoapparat ihn herstellt“ übersetzt werden kann, nimmt das Motiv der Analyse durch Sistierung eines optisch Unbewussten auf. Die experimentelle Anordnung chronofotografischer Beobachtung zerlegt die Bewegung in einzelne - von den Wissenschaftlern zu analysierende - Segmente und eröffnet damit zugleich die Möglichkeit zur Synthetisierung in der Illusion des sich bewegenden Bildes. Versuchspersonen laufen, springen und steigen Treppen, in Laboratorien wie in den dokumentarischen Filmen, in ethnografischen ebenso wie in fiktionalen Settings. Ihr Verhalten teilt sich entsprechend paradigmatischer Unterscheidungen mit: normal oder anormal, soldatisch oder pathologisch. Berechnet, verrechnet und abgerechnet wird, nachdem die Bewegungen grafisch in Fotoplatten eingeschrieben sind. „Die aufzeichnenden Geräte messen die Infinitesimalen der Zeit; die schnellsten und die schwächsten Bewegungen, die geringsten Änderungen der Kräfte können ihnen nicht entgehen. Sie dringen in die intimsten Funktionen der Organe ein, wo sich das Leben als ständige Mobilität zu vermitteln scheint.“"16

Bittet in Westworld der Gründer der Firma, Dr. Robert Ford (gespielt von Anthony Hopkins), die hosts zum Gespräch, so werden auch hier keine Schädel mehr geöffnet. Apathisch und ihres Ausdrucks beraubt, sitzen die Androiden vor ihrem Schöpfer und beantworten Fragen. Das psychoanalytische Setting Freuds wird modifiziert, der Analytiker verschwindet nicht im Rücken des Analysanden; er sitzt ihm gegenüber. Nicht Körpervergessenheit und die Rede eines Unbewussten, sondern Zentrierung des Körpers und das Lesen der Mimik und Gestik verraten, ob ein host nach - oder eben

16 Étienne-Juley Marey: Die graphische Methode in den experimentellen Wissenschaften (1878). In: B. Schneider, C. Ernst, J. Wöpking (Hg.): Diagrammatik-Reader. Grundlegende Texte aus Theorie und Geschichte, Berlin/Boston 2017, S. 203-206, S. 204. 
nicht nach - Protokoll operiert. Es gibt keinen Einblick in die Schaltkreise, die ganz im Sinne der Kybernetik black boxes bleiben. Jedoch werden die hosts energetisch nachgeladen, mithilfe von Computern und mobilen Touchpads werden Stromkreise neu verdrahtet und Spannungen verändert. Auch das geht zurück auf Laborgeschichten. 1949 konstruierten Norbert Wiener und Henry Singleton eine heliotrope Maschine, die sich mithilfe einer internen Steuerung auf Licht zubewegen konnte, wie eine Motte, oder vor dem Licht verkriechen, wie eine Wanze. Die Palomilla war mit technischen Sinnesorganen ausgestattet, die die Lichtintensität aus der Umwelt aufnahmen und als Information, also Signal weitergaben an eine schwachstromgespeiste Steuerung, die ihrerseits die mit Starkstrom betriebenen motorischen Teile lenkte. Wenn sich das Gerät bewegte, änderte sich wiederum die Ausrichtung seiner sensorischen Elemente und steuerten so, je nach Position, die Bewegungsmotorik neu aus. So simulierte die Palomilla als eines der ersten künstlichen Lebewesen Verhalten im integrierten Kreislauf. Das Prinzip der Rückkopplung, das zugunsten eines einfachen und vergleichenden Informationstransfers auf Konzepte von Innen und Außen verzichten kann - ließ sich nur und unmittelbar an der wechselseitigen Ausrichtung von Individuum und Milieu erkennen. „Mit Verhalten ist jede Veränderung einer Entität in Bezug zu ihrer Umgebung gemeint. [...] Dementsprechend, kann jede Veränderung eines Objekts, die von außen wahrgenommen werden kann, als Verhalten bezeichnet werden. ${ }^{17}$ Die Rückkopplung, die entweder erhaltend - homöostatisch - oder korrigierend funktioniert, folgt Impulsen aus der Umwelt und wirkt dann wieder auf diese ein. Ein Philosoph wie Max Bense kann daher in seiner Beschreibung solcher Interaktionen dem älteren Paradigma verhaftet bleiben und von einem „reagierenden Bewußtsein“18 sprechen um festzustellen, dass Perfektion reine Automatik, aber auch das Ende aller Entwicklung bedeutet. Imperfektion, Brüche, Fehlschaltungen und Störungen erst machen Verhalten aus, machen unheimliche Apparate und Apparate unheimlich - aber eben auch optimierbar.

Die Experimente am MIT in den fünfziger Jahren, die Prinzipien sensomotorischer Rückkopplung als gegenseitige Regelung von Wahrnehmung und Bewegung, neuronalen und motorischen Prozessen erforschten, reduzierten freilich das Verhältnis von Eingabe und Ausgabe von Signalen in maschinellen oder menschlichen Kreisläufen

17 „By behavior is meant any change of an entity with respect to its surroundings. [...] Accordingly, any modification of an object, detectable externally, may be denoted as behavior." Arturo Rosenblueth, Norbert Wiener, Julian Bigelow: Behavior, Purpose and Teleology. In: Philosophy of Science, Jg. 10, 1943, S. $18-24$, S. 18.

18 Max Bense: Kybernetik oder die Metatechnik einer Maschine. In: Max Bense. Ausgewählte Schriften, Bd. 2: Philosophie der Mathematik, Naturwissenschaft und Technik, Stuttgart 1998, S. 429-446, S. 444. 
auf wenige grundlegende Parameter. Doch bereits damit ließ sich im Verhalten simulieren, was überaus menschlich erschien: Palomilla, das tropismusgesteuerte Räderviech, zeigte zur Überraschung des Mathematikers Wiener und des Mediziners Rosenblueth, dass nicht nur Orientierungen im Raum, sondern auch Pathologien dieser Orientierung anhand der Regelung der Rückkopplungsmechanismen sichtbar wurden: War die Übertragung der Signale zu stark eingestellt, zeigte die Palomilla ein Verhalten, das exakt den motorischen Übersteuerungen glich, wie sie als Intentionstremor bekannt waren. War die Rückkopplung zu schwach eingestellt, verhielt sich die Palomilla orientierungslos und selbstvergessen wie ein Parkinsonpatient - oder wie host Vater in Westworld.$^{19}$ Diese erste sehr primitive, aber doch in ihrem spezifischen Verhalten charakterisierbare Maschine wurde bald durch ein mechanisches Tier von Grey Walters übertroffen, das durch statistische Vorhersagen aufgrund gleichbleibender Handlungsabläufe das Muster seines gesamten Verhaltensschemas ändern, also lernen konnte. Für Wiener war damit klar, dass Verhalten Kommunikation ist und jeder Mensch eine Nachricht, die mit Kommunikationsmaschinen der Zukunft verbunden werden kann. ${ }^{20}$

Im Konzept des Verhaltens, das durch die Rückkopplung von Eigenem und Fremden, von Menschenkörper und technischer Anordnung bewusst wird, begegnen sich die Geschichte der Kybernetik, die Geschichten des Kinos und ein phänomenologisches Konzept des Bewusstseins:

\begin{abstract}
„Bewusstsein ist einmal ein besonderer, und zwar fundierter Verhaltenstyp und mithin ein Sonderbereich, zum anderen ist es das, universale Milieu', in dem sich die Strukturen und Verhaltensweisen darstellen und voneinander abheben. Die Blickwende, die im Gesehenen das Sehen und den Sehenden selbst vor Augen führt, ist ein Akt der Reflexion." ${ }^{21}$
\end{abstract}

Die Logik des Zirkulär-Kausalen also liegt allem Verhalten zugrunde. Die französische Schule der Phänomenologie und vor allem Maurice Merleau-Ponty haben das als Dialektik des Verhaltens bezeichnet. Merleau-Ponty konnte sein Konzept des comportement daher zugleich auf eine technisch-biologische Geschichte der Kybernetik zurückführen und auf die Wahrnehmungsgeschichte des Kinematografischen selbst.

Für Robert Ford in Westworld ist, genau wie einst für Norbert Wiener, die Möglichkeit eines malfunctioning von kybernetischen Intelligenzen, Lebewesen und Maschi-

19 Norbert Wiener: Mensch und Menschmaschine, Frankfurt a. M./Berlin 1952, S. 173ff.

20 Wiener: Mensch (s. Anm. 19).

21 Bernhard Waldenfels: Vorwort des Übersetzers. In: Maurice Merleau-Ponty: Die Struktur des Verhaltens, Berlin/New York 1976, S. V-Xxi, S. XVII. 
nen einerseits bedrohlich, andererseits der deutlich interessantere Teil der Forschung. „Was tun wir bei einer Maschine, wenn ein solcher Unfall auftritt“, fragte Wiener. ${ }^{22}$ Seine Reparaturvorschläge markieren das gesamte Spektrum dessen, was auch in der Westworld zu sehen ist: „Zuerst bemühen wir uns, sämtliche Informationen zu löschen, in der Hoffnung, dass die Schwierigkeit bei einem Neustart mit anderen Daten nicht

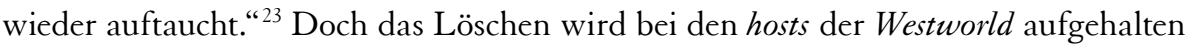
durch eben jene reveries, die alte Programmierungen reaktivieren, in den Arbeitsspeicher holen, ins Gedächtnis rufen. Eine Maschine lässt sich in solchen Fällen auch, schreibt Wiener, gut schütteln - wie es ja Eltern zum Teil auch mit ihren Kindern versuchten - oder einem starken elektrischen Impuls aussetzen, wie die Ingenieure in der Serie es tun. Die sanftere, aber nicht sehr effektive Methode einer guten Reinigung des Gehirns sei für Menschen der Schlaf, der allerdings die Erinnerungen nicht löscht. Dass der Vater von Dolores, Peter, nicht mehr schläft, ist daher auch im kybernetischen Sinne ein höchst beunruhigendes Symptom. In Fällen endlos oszillierender Daten im Kreislauf, merkt Wiener kritisch an, werden häufig ,wesentlich brutalere Eingriffsmethoden in den Gedächtniskreislauf" vorgenommen, die Lobotomie, die die Fähigkeit zerstört, ,sich jene anhaltenden Sorgen zu machen, die in der Terminologie eines anderen Berufsstandes Gewissen genannt werden“ “. ${ }^{24}$ Genau solche Varianten der kybernetischen Therapie werden im Verlauf der ersten Staffel der Filmserie verhandelt: den hosts wird die Energie entzogen, Nervenverbindungen werden durchtrennt und neu verlötet, sie werden kaltgestellt oder von anderen Figuren geschüttelt oder - westernstyle - verprügelt.

\section{Rückkopplung als Wissen der Anderen}

Die gegenwärtige Kultur des Ich, die, als eine Art $i K u l t$, alle Verbindungen zu ihrer Genealogie in der Utopie von Kontrolle und Kommunikation kybernetischer Forschungen zumindest terminologisch zu kappen trachtet, behauptet sich in medizinischen und ästhetischen ebenso wie in kulturindustriellen Kontexten als Triumph des Individuums in seinem durch technische Extension oder Substitution nahtlos zu optimierendem Körper. Dieser Triumph verstellt jedoch den Blick darauf, dass es weniger der homo protheticus ${ }^{25}$ in seiner vermeintlichen Kohärenz ist, für den sich Biopolitik zu

22 Norbert Wiener: Kybernetik (1948). In: Bernard Dotzler (Hg.): Norbert Wiener. Futurum Exactum. Ausgewählte Schriften zur Kybernetik und Kommunikationstheorie, Wien/New York 2002, S. 13-29, S. 22.

23 Wiener: Kybernetik (s. Anm. 22).

24 Wiener: Kybernetik (s. Anm. 22), S. 23.

25 Vgl. Karin Harasser: Körper 2.0. Über die technische Erweiterbarkeit des Menschen, Bielefeld 2013. 
interessieren hätte, als vielmehr die Vernetzung von Körpern oder, genauer, Körperteilen in Relais technischer Gadgets und ihrer Schnittstellen, zu denen nicht zuletzt auch das Post-Kinematografische in all seinen neuen digital organisierten Oberflächen gehört. Damit werden alle Körper durch ihr Verhalten zuerst Körper für andere, hosts im strengen Sinne des Gastgebers als Datengeber, und damit erst Ich oder $i$ Ding für uns selbst. Die Dialektik des corps propre Merleau-Pontys verweist darauf ebenso wie die strukturale Psychoanalyse Jacques Lacans. Beide verhandeln damit auch Überlegungen aus der frühen Kybernetik, die sich der Herausforderung stellte, Verhalten nicht länger als etwas zu untersuchen, das sich an individuellen und persönlichen Zielen und Zwecken ermessen lässt, sondern dessen Formen und Richtungen sich als Resultat ständiger Rückkopplungen mit der Umwelt und ihrer Systeme erkennen lassen. ${ }^{26}$ Inzwischen ist diese Logik der Nachbarschaften als solche der Schwärme und ihrer Intelligenzen etabliert und als Grundsatz in der Bildung von Managements oder Smart Mobs eingesetzt. ${ }^{27}$ In ihren kybernetischen Anfängen in den vierziger Jahren des 20. Jahrhunderts jedoch war die Annahme eines ungerichteten, nicht-teleologischen Verhaltens, das sich lediglich an den Strukturen seiner Umgebung orientiert, wie Gregory Bateson anmerkt, absolut rätselhaft, insofern „Zweck vollkommenes Mysterium war“ ${ }^{28}$ Dabei hieß das zunächst nur, dass sich eine Logik des Verhaltens nicht mehr aus einer Seele des Handelnden erklären ließ, sondern aus einem Verhältnis zu Umwelt:
„Der Begriff der Zweckgerichtetheit soll kennzeichnen, dass eine Handlung Akt oder ein Verhalten interpretiert werden kann auf das Erreichen eines Ziels hin ori- entiert - d.h., auf einen finalen Zustand, in dem das [sich] verhaltende Objekt eine endgültige Beziehung in Zeit oder Raum in Verhältnis zu einem anderen Objekt oder Ereignis erreicht. ${ }^{\text {29 }}$

Margaret Mead berichtet, dass Anthropologen und Anthropologinnen gegen die Mathematikerinnen und Ingenieure unter den Kybernetikern, die sich lieber auf Gesetze großer Zahlen verlassen wollten, in der Bestimmung des Verhaltens das

26 Rosenblueth, Wiener, Bigelow (s. Anm. 17).

27 Eugene Thacker: Netzwerke - Schwärme - Multitudes. In: Eva Horn, Lucas Marco Gisi (Hg.): Schwärme. Kollektive ohne Zentrum. Eine Wissensgeschichte zwischen Leben und Information, Bielefeld 2009, S. 27-68.

28 „Purpose was a total mystery.“ Gregory Bateson, Margaret Mead, Stewart Brand: For God's Sake Margaret. In: CoEvolutionary Quarterly, June 1976, Nr. 10, S. 32-44, S. 38.

29 „The term purposeful is meant to denote that the act or behavior may be interpreted as directed to the attainment of a goal - i. e., to a final condition in which the behaving object reaches a definite correlation in time or in space with respect to another object or event.“ Rosenblueth, Wiener, Biogelow (s. Anm. 17), S. 18. 
Prinzip des Musters gegen das Kalkül der großen Zahl durchsetzen mussten: pattern against purpose. Heute ist es genau das, was die Kinder von den hosts lernen: sich in Musterumgebungen sehr schnell zu bewegen und bewegen zu lassen.

Inzwischen sind die Schnittstellen und Interfaces, die haptischen und taktilen ebenso wie die stimmlichen und sprachlichen Übertragungen, mit denen Norbert Wiener am MIT experimentierte, verfeinert und an potentere Datenspeicher und -prozessoren angeschlossen, die in der Tat nicht nur erhöhte Wirklichkeiten des Erinnerns, Vorstellens und Reagierens realisiert haben, sondern buchstäblich eine augmented reality des Menschlichen insgesamt. Nicht dass Computer ein Bewusstsein entwickeln, wie Ray Kurzweil es schon 1999 für die nahe Zukunft prognostizierte, sondern dass Bewusstsein überhaupt nur noch als Relation von Mensch und Maschine gedacht werden kann, fordert das Denken heraus:

„An der Stelle, wo die Philosophie eine ursprüngliche, Vertrautheit-mit-uns-selbst' lehrt, die erstens Abbildungsbeziehungen als solche wahrnehmen und zweitens einen ,kontinuierlichen Iterationsprozeß' (M. Frank) speichern kann, so daß alle Individuen im Erzählen ihrer Lebensgeschichte kleine Autoren oder Goethes werden, an genau dieser Stelle insistiert die Psychoanalyse darauf, daß Bewußtein nur die imaginäre Innenansicht medialer Standards ist. ${ }^{\text {" }} 0$

Im historischen Moment, an dem diese Ununterscheidbarkeit von menschlichem und nichtmenschlichem Anteil in Denk- und Erinnerungsprozessen ebenso wie im Verhalten, etwa im Straßenverkehr unter Bedingungen selbststeuernder Autos aufmerksame und zugleich ratlose Juristinnen und Juristen aufruft, Fragen von Willen und Intention, Verantwortung und Recht neu zu verhandeln, ${ }^{31}$ interveniert die schlaue Neuauflage von Westworld als Imagination einer Welt der Pioniere im rechtlosen frontier-Gebiet. Darin beschreibt sie eine Umkehr der US-amerikanischen Geschichte in eine Geschichte der THEM. Mit diesen großgeschrieben Anderen sympathisieren die Androiden, wenn sie sich gegen das US, das phantasmatische WIR wenden, sich besinnen und aufbegehren.

Westworld legt das Verhältnis von Körper und Technologie unter Bedingungen eines digital ver- und berechnenden Milieus offen. Damit schließt die Serie an die Wissenschaft der Kybernetik und an die Phänomenologie an, welche es ins Zentrum ihrer Theorien gestellt haben. Deutlich nimmt Westworld zahlreiche Motive des Westerns

30 Kittler: Die Welt des Symbolischen (s. Anm. 11), S. 61.

31 Vgl. Sabine Gless, Kurt Seelmann (Hg.): Intelligente Agenten und das Recht, Baden-Baden 2016; Ryan Calo, A. Michael Froomkin, Ian Kerr: Robot Law, Cheltenham 2016. 
oder frontier-Films auf: die Ausdifferenzierung von Natur und Kultur, von Gewalt und Gesetz, Fortpflanzung und Sinnlichkeit, Männern und Frauen. Alle diese Unterscheidungen erscheinen als Verhalten, wie US-amerikanische Popkultur es vor allem durch das Kino globalisiert hat. Darüber hinaus aber bleibt die Serie vor allem deshalb eine irritierende Erfahrung, weil sie die Laboratorien, in denen Menschen künstlich gemacht werden, nicht nur zeigt und in ihren historischen Geräten und Verfahren aufruft, sondern weil die Serie selbst in ihrer Ästhetik und netzwerkartigen Distribution ein Laboratorium herstellt, das die Frage nach einer künstlichen Implementierung von Bewusstsein als datengesteuertes Verhalten mit jeder Szene, mit jedem Schnitt aufwirft. Westworld zeigt damit auch, dass eine Differenzierung von inner-, außeroder transdiegetischer Welt unter Bedingungen universeller und dennoch agonaler Datenprozessierung nicht mehr sinnvoll ist. Westworld macht deutlich, inwiefern jede und jeder unter Netzwerkbedingungen Datenspuren hinterlässt, die das Verhalten aller anderen und damit schließlich auch das eigene modifizieren. In integrierten Schaltkreisen wird jede und jeder durch Verhalten zur Nachricht für alle anderen, die sich einschalten und zuschalten, weil sie sich dafür interessieren: to whom it may concern. ${ }^{32}$ Bereits Norbert Wiener hat das als Struktur der universalen message bezeichnet. Unser Verhalten, das sich immer dank irgendwelcher Gadgets und immer über irgendwelche Monitore auf andere überträgt, generiert mit allen Botschaften zugleich Daten, von denen wir, algorithmisch unterbelichtet, nicht wissen, welche Gespenster am Wegesrand von Datenautobahnen und click-farms sie abzapfen. Welche hosts nehmen unser Verhalten an? Können wir aufbegehren gegen programmiertes Verhalten? Sich so zu benehmen, dass das Muster des Verhaltensschemas mit jeder Handlung fundamental reorganisiert und auch die Umgebung neu geordnet wird, das ist der kategorische Imperativ, den wir von den hosts der Westworld lernen können.

32 Vgl. Erhard Schüttpelz: To Whom it May Concern Messages. In: Claus Pias (Hg.): Cybernetics/Kybernetik. Die Macy Konferenzen 1946-1953, Bd. 2: Essays \& Dokumente, Berlin/Zürich 2004, S 183-198. 